\title{
Multiple visceral resections for pelvic recurrence from surgically treated clear cell ovarian tumor - a case report
}

\author{
Irina Bălescu ${ }^{1}$, Nicolae Bacalbaşa ${ }^{2}$, Radu Zamfir ${ }^{3}$, Dana Tomescu ${ }^{4}$, Vladislav Braşoveanu ${ }^{3}$ \\ Corresponding author: \\ Nicolae Bacalbaşa, MD \\ No. 2, Dimitrie Racovită Street, \\ Bucharest, Romania \\ E-mail: nicolae_bacalbasa@yahoo.ro \\ 1"Ponderas" Hospital, Bucharest, Romania \\ 2"Carol Davila" University of Medicine and Pharmacy, Bucharest, Romania \\ 3"Dan Setlacec" Center of Gastrointestinal Disease and Liver Transplantation, \\ "Fundeni" Clinical Institute, Bucharest, Romania \\ ${ }^{4}$ Department of Anaethesia and Critical Care III, Fundeni Clinical Institute, Bucharest, Romania
}

\begin{abstract}
Ovarian clear cell carcinoma is a distinct histopathological subtype of epithelail ovarian cancer with a more aggressive biological behaviour and a higher capacity to recur. We present the case of a 47 year old patient diagnosed with a clear cell ovarian tumor invading an ileal loop submitted to radical surgery followed by adjuvant chemotherapy. At one year follow up she was diagnosed with a pelvic recurrence invading the right ureter and the right ileocolon so the patient was re-submitted to surgery. The recurrence was resected en bloc with the right ileocolon and distal ureterectomy. The continuity of the digestive tract was re-established bya ileo-transverse anastomosis while the right ureter was re-inserted in the urinary bladder. The postoperative course was uneventful.
\end{abstract}

Key words: clear cell ovarian tumor, pelvic recurrence, multiple visceral resections

\section{INTRODUCTION}

Clear cell carcinoma of the ovary is considered as a distinct histopathological type of ovarian tumors accounting for up to $5 \%$ of all ovarian epithelial malignancies (1). Patients diagnosed with this tumor subtype usually report a poor prognosis, this histopathological subtype being considered as a more biologically aggressive tumor with minimal chemotherapy responsiveness $(2,3,4)$. Even in cases with limited stage disease treated by surgery and adjuvant chemotherapy recurrence will develop in up to one third of cases $(1,5)$.

\section{CASE REPORT}

A 47-year-old patient was initially investigated for pelvic pain and subocclusive syndrome. The imagistic studies revealed the presence of a pelvic tumor with possible ovarian origin invading an ileal loop so the patient was submitted to surgery; intraoperatively a large retro-uterine tumor invading the 
right adnexa and an ileal loop located at $50 \mathrm{~cm}$ from the ileo-caecal valve was found; the tumor was resected en bloc with total hysterectomy, bilateral adnexectomy and segmental ileal resection; the continuity of the digestive tract was re-established by an end to end anastomosis. The histopathalogical study revealed the presence of a poorly differentiated adenocarcinoma but could not distinguish between a digestive neuroendocrine tumor and a clear cell ovarian carcinoma. The immunohistochemical studies confirmed the presence of diffuse positive Ck7, CA 125 and oestrogen receptors, with negative tests for chromogranine and synaptophisine so the final diagnosis was the one of a poorly differentiated clear cell ovarian tumor. Ki 67 was positive in up to $80 \%$ of the tumoral cells. The patient was referred to the oncology clinic where she was submitted to six cycles of adjuvant chemotherapy with platinum salts and taxanes regimens. One year after ending the adjuvant treatment the patient was diagnosed with a pelvic recurrence invading the right colon, the right hypogastric artery, the wall of the right external iliac vein and the right ureter (figures 1, 2). The patient was re-submitted to surgery and the recurrence was resected en bloc with right ileo-colectomy and distal ureterectomy. The right hypoasgtric artery was ligated while the invaded area od the right external iliac vein was laterally resected and sutured (figures 3-8). The continuity of the digestive tract was re-established by an side to end ileo-transverse anastomosis while the right ureter was re-implanted in the urinary bladder (figure 9). The urinary anastomosis was protected by placing a double J urinary catheter which was removed 3 weeks later. The postoperative course was uneventful, the patient being discharged the $11^{\text {th }}$ postoperative day. The histopathological study confirmed the presence of a poorly differentiated clear cell adenocarcinoma with ovarian origin.

\section{DISCUSSIONS}

Clear cell ovarian carcinoma represents up to $5 \%$ of all types of ovarian cancer and is usually associated with a poorer prognosis when compared to the serous subtype (2). At the time of diagnosis most patients present with a pelvic mass while the initial stage at diagnosis widely varies. It has been reported that $57-$ $81 \%$ of cases are diagnosed in an early stage of the disease (6-8). In order to better understand the molecular basis of the disease and the most important prognostic factors a group of researchers reviewed the clinical, pathological and therapeutical features of clear cell ovarian carcinoma in 2010 in Vancouver (8). It has

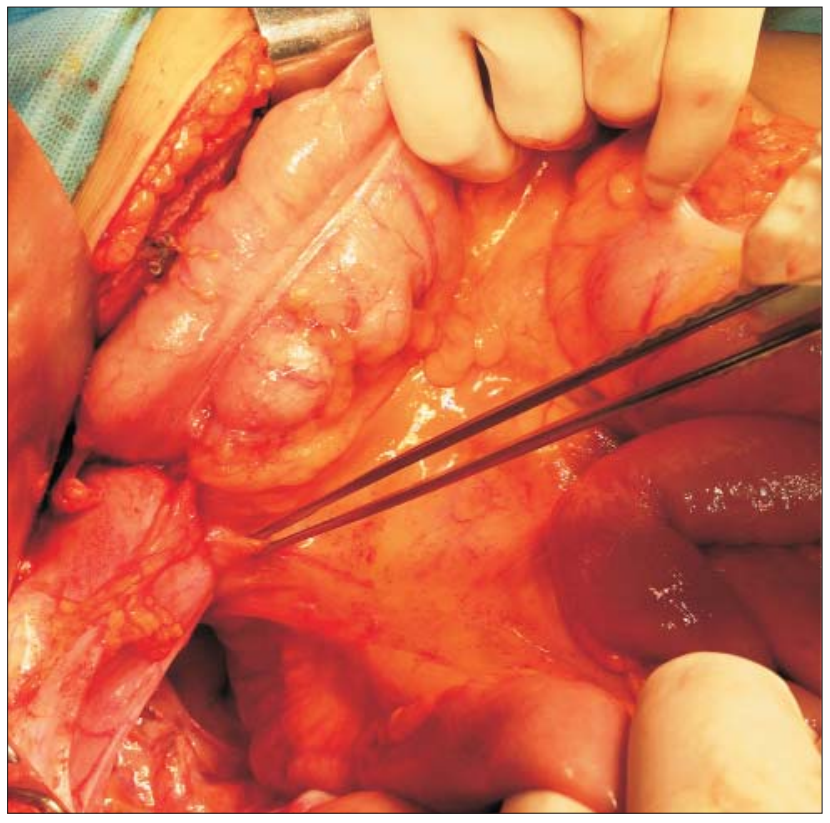

Figure 1 - Initial aspect - large pelvic recurrence invading the right colon

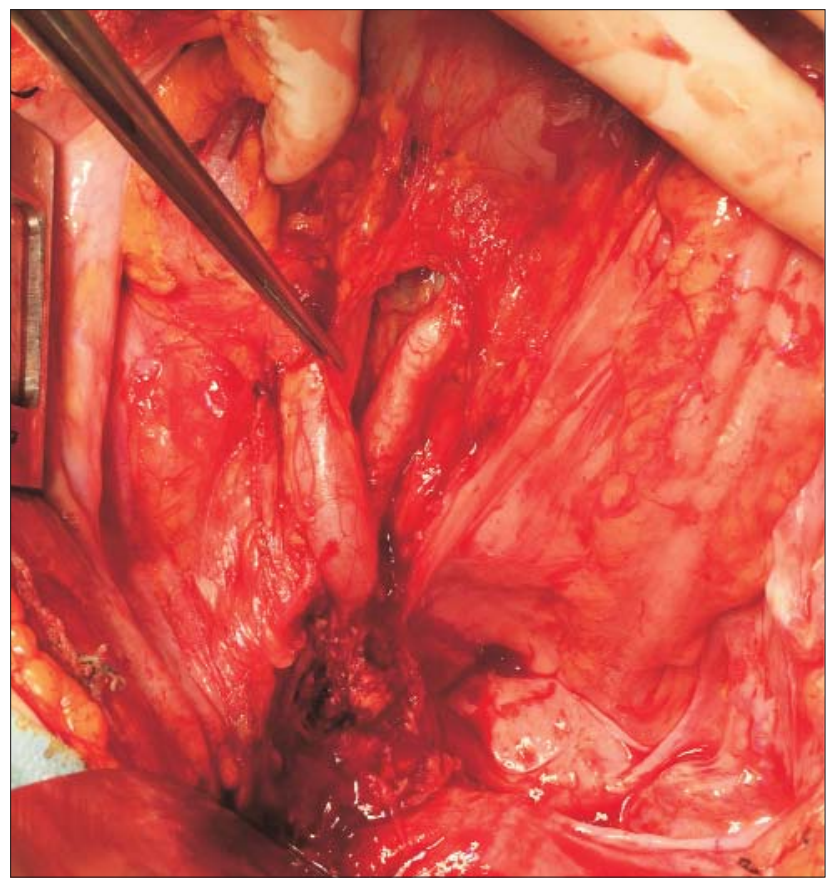

Figure 2 - The pelvic recurrence is invading the right ureter

been demonstrated that clear cell ovarian carcinoma have the highest rate of PIK3CA mutations among all types of ovarian cancer and has been stipulated that PI3K-AKT-mTOR-HIF (phosphoinositide 3-kinase, v-akt murine thymoma viral oncogene homolog, mechanistic target of rapamycin, and hypoxia induced factor) pathway may be a therapeutically viable target (9-13). Other similarities encountered between 


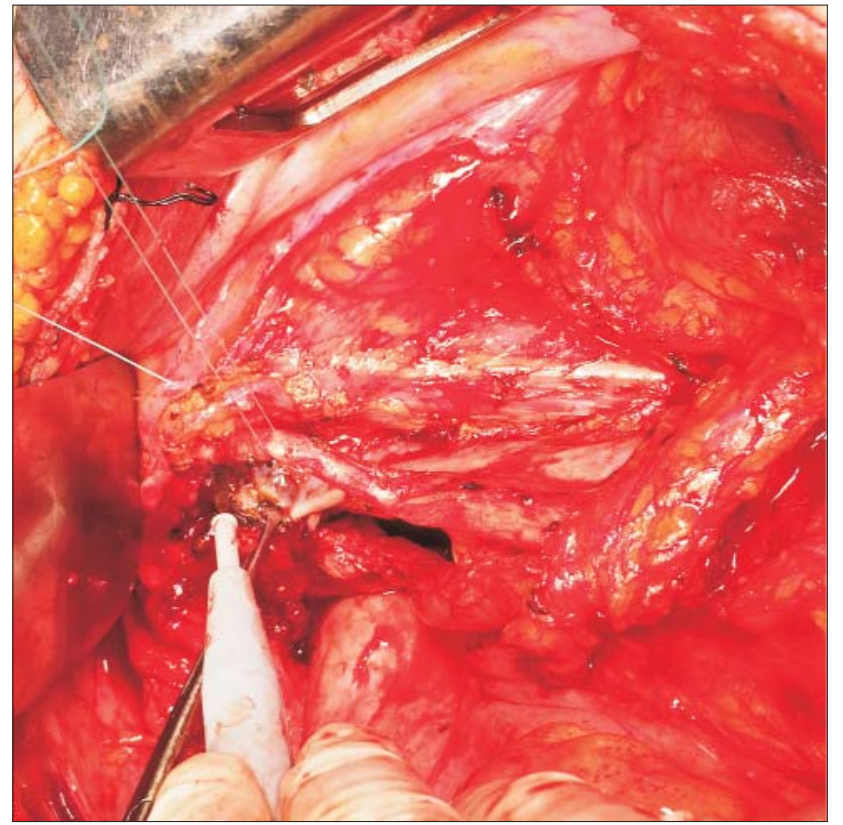

Figure 3 - Dissection of the right iliac artery

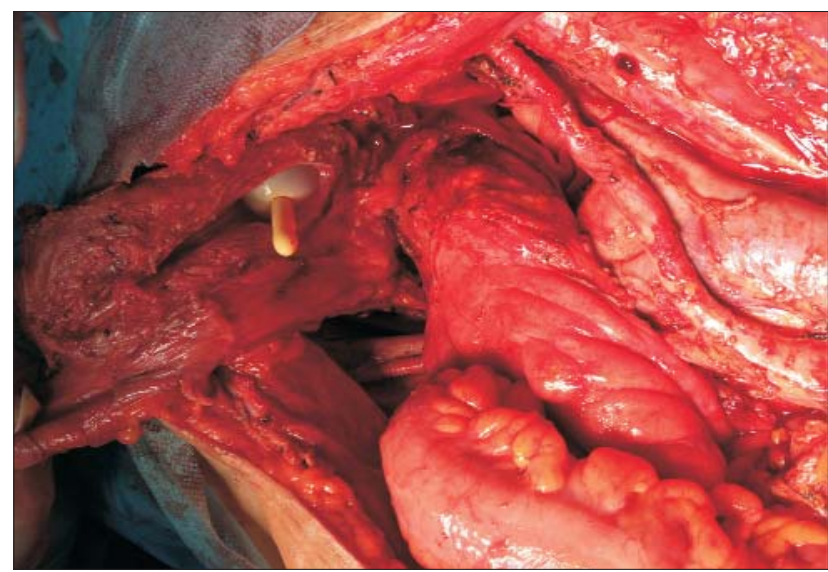

Figure 5 - The final aspect after mobilization of the tumor en bloc with the invaded urinary bladder

ovarian clear cell carcinoma and clear cell tumors with other locations such as renal or endometrial tumors have been described and therefore possible common therapies have been proposed (14). However, despite many similarities between renal and ovarian clear cell carcinomas, they practically represent different diseases; patients diagnosed with ovarian CCC do not present somatic and germline mutations in the VHL gene while in those with renal CCC these are commonly seen (8).

When it comes to cases diagnosed in an advanced stage of the disease, surgery seems to be the most appropriate therapy especially due to the fact that clear cell ovarian carcinoma is less responsive to chemotherapy and patients who have a poor response to first

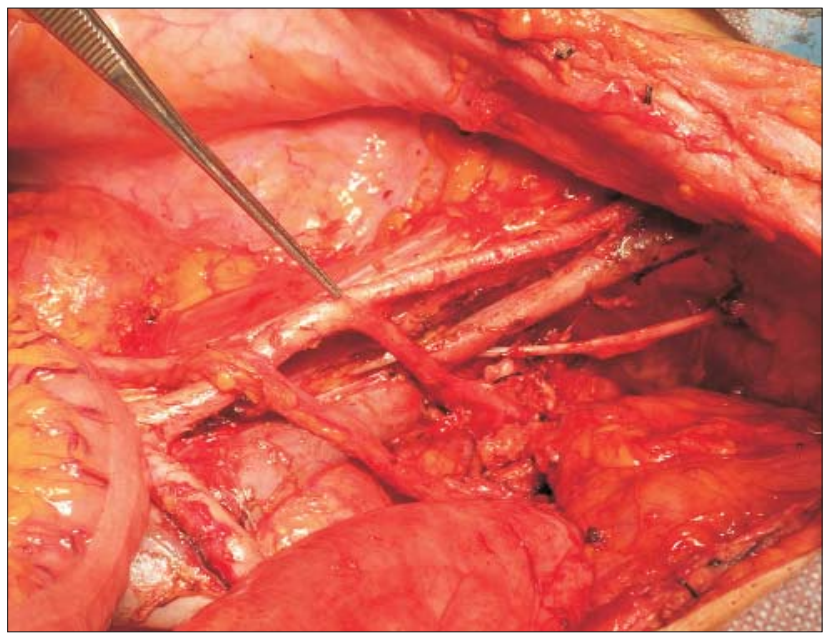

Figure 4 - Dissection of the left obturatory fossa

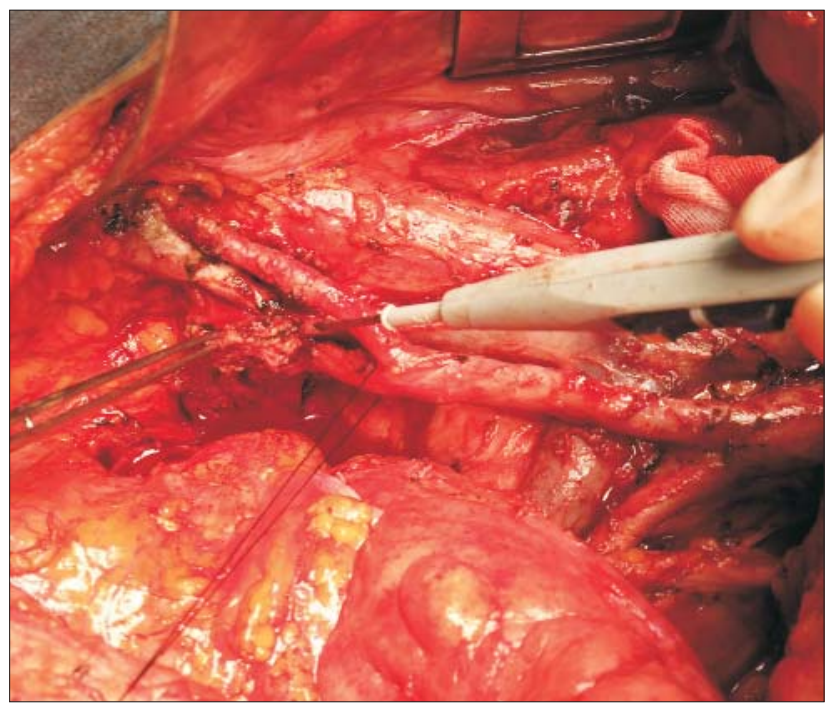

Figure 6 - The aspect of the right obturatory fossa after ligation of the right hypogastric artery. Infiltration of the venous wall of the right external iliac vein

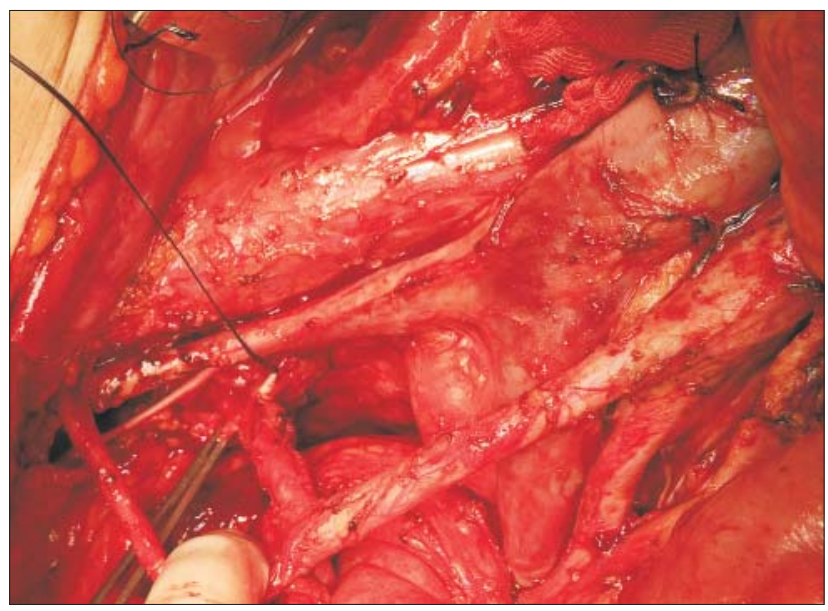

Figure 7 - The aspect after resection of the invaded venous wall. The right external iliac artery is completely dissected 


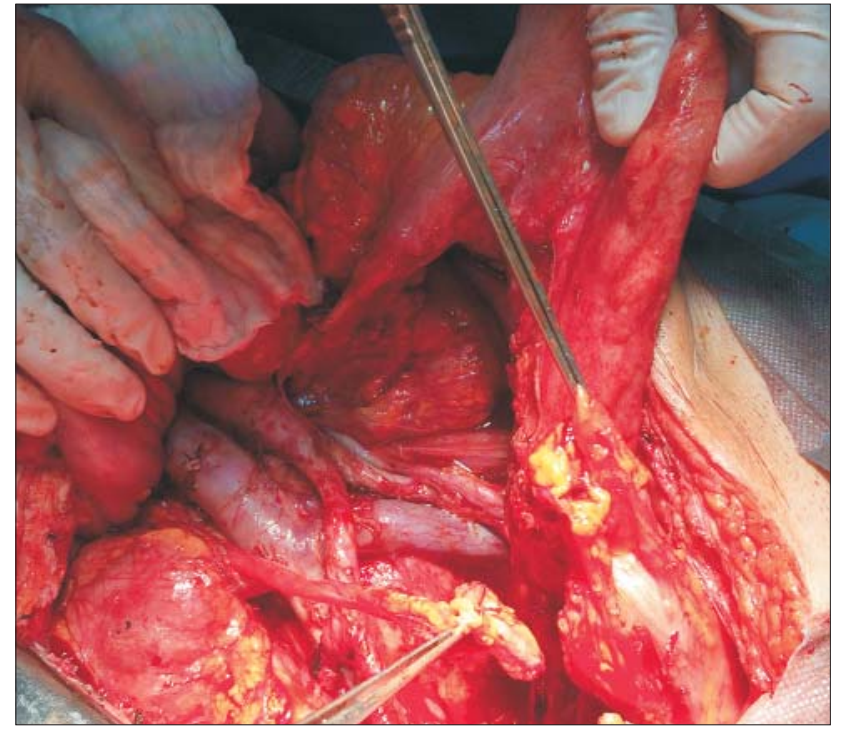

Figure 8 - Preparing the right ureter for re-implantation

line chemotherapy are resistant to second-line therapy. The poor response to chemotherapy is mainly related to ovarian CCC's platinum resistance; Sugiyama et al reported that the response rate to platinum and taxanes therapy is significantly lower for patients with CCC when compared to those with epithelial ovarian cancer $911 \%$ versus $72,5 \%)(6)$. Due to this fact cytoreduction should be performed in all cases and ovarian CCC should be managed in the same manner as epithelial ovarian cancer (1, 15-17). However, given the rarity of cases, the role of surgery has not been prospectively evaluated (18).

In order to determine the most important prognostic determinants for patients diagnosed with gynaecological CCC, Rauh-Hain et al conducted a study on 5421 women with ovarian and uterine CCC; patients data were found in 18 SEER registries between 1988-2010. Advanced stage ovarian CCC with regional involvement was found in 398 cases (11\% of patients) while distant involvement was seen in 1411 cases (38.,9\%). Among patients with regional disease, 5-year disease free survival was $65.8 \%$ for ovarian CCC; in contrast, the 5 year cancer-specific survival was $57.8 \%$ $(53.1 \%-62.8 \%)$ for women with uterine CCC ( $p=0.01)$. The Cox proportional hazards model identified an independent association of older age, African-American race, SEER registry location, advanced stage, absence of surgical treatment, and absence of lymph node dissection with cancer-specific mortality. The Cox model demonstrated that the strongest quantitative predictor of death was stage at the time of diagnosis, with more advanced stages associated with higher rates of mortality. Although in univariate analysis patients

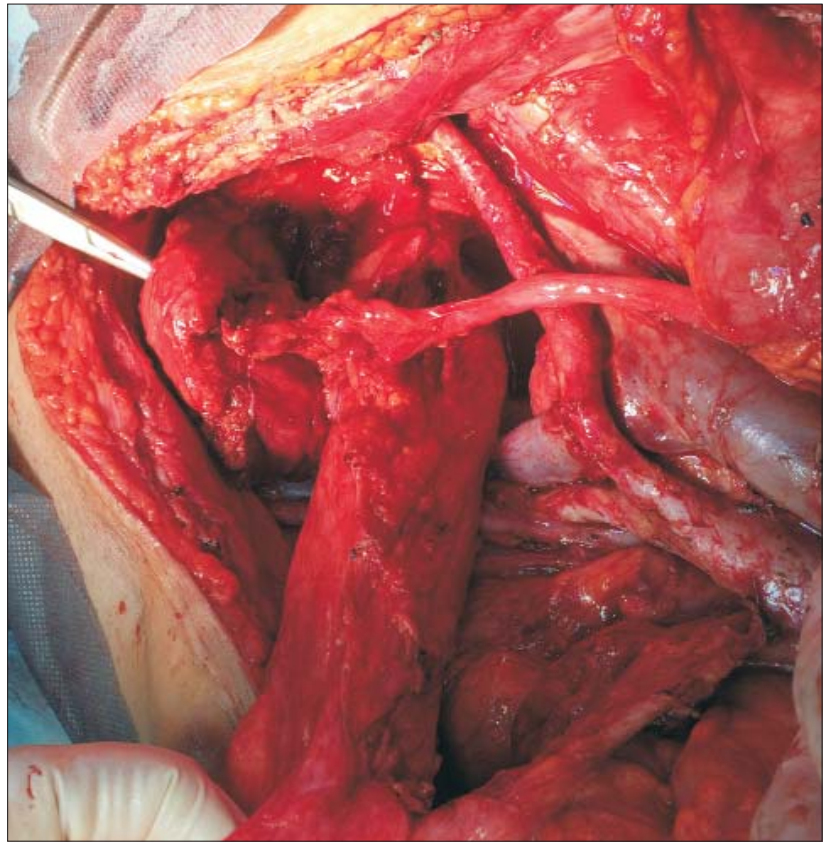

Figure 9 - Reimplantation of the right ureter

diagnosed with advanced stage ovarian CCC had a better outcome, in multivariate analysis there was no significant difference in terms of survival between cases with locally advanced ovarian or uterine CCC (19).

In their meta-analysis, Lee et al studied the long term outcomes of ovarian CCC compared to other histological subtypes. When comparing the hazard ratio based on stage I-II and stage III-IV patients with ovarian CCC had a higher hazard arte for death among all stages when compared to those with non-CCC. When focusing on the differences between advanced stage ovarian CCC and advanced stage epithelial ovarian cancer, a poorer outcome was seen in cases with ovarian CCC. When studying the outcomes of CCC compared to each histopathological subtype of epithelial ovarian cancer, a better outcome was observed for endometroid epithelial ovarian cancer; this difference was not maintained when it came to mucinous epithelial ovarian cancer.

When it comes to recurrent ovarian CCC, it seems that it is practically unresponsive to chemotherapy. In the study conducted by Levenback et al involving 51 patients with recurrent ovarian CCC 105 regimens were administrated. At the time of recurrence, among patients with platinum sensitive disease only $9 \%$ had partial response to re-treatment and $18 \%$ had stable disease while among cases with platinum resistant disease only $1 \%$ reported a partial response to gemcitabine (20).

In the study conducted by Kajiyama et al regarding post-recurrent oncologic outcome of patients with 
ovarian CCC, both overall survival and post-recurrence survival were significantly shorter for patients with ovarian CCC when compared to those with recurrent serous adenocarcinomas (21).

\section{CONCLUSIONS}

Ovarian CCC represent an aggressive biological subtype of ovarian cancer associated with poor prognosis and low rates of long term survivors. Although due to the paucity of reported cases a standard therapeutic protocol has not yet been established it seems that cytoreductive surgery is the only potential curative solution especially due to the high chemoresistence developed by these patients. When it comes to recurrent tumors, they seem practically unresponsive to chemotherapy, so re-resection is perfectly justified in order to prolong survival.

\section{Acknowledgement}

This paper was co-financed from the European Social Fund, through POSDRU 2007-2013, Prioritary axis no. 1, „Education and formation supporting economical development and aknowledgment based society development- major domain of intervention 1.5 "Doctoral and post-doctoral programs supporting research".

CERO - carrier profile: - Romanian researcher

Financial contract: POSDRU/159/1.5/S/135760

\section{REFERENCES}

1. Pather S, Quinn MA. Clear-cell cancer of the ovary-is it chemosensitive? Int J Gynecol Cancer 2005 May;15(3):432-7.

2. Goff BA, Sainz dIC, Muntz HG, Fleischhacker D, Ek M, Rice LW, et al. Clear cell carcinoma of the ovary: a distinct histologic type with poor prognosis and resistance to platinum-based chemotherapy in stage III disease. Gynecol Oncol 1996 Mar;60(3):412-7.

3. Recio F0, Piver MS, Hempling RE, Driscoll DL. Lack of improved survival plus increase in thromboembolic complications in patients with clear cell carcinoma of the ovary treated with platinum versus nonplatinum-based chemotherapy. Cancer 1996 Nov 15;78(10):2157-63.

4. Al Barrak J, Santos JL, Tinker A, Hoskins P, Gilks CB, Lau H, et al. Exploring palliative treatment outcomes in women with advanced or recurrent ovarian clear cell carcinoma. Gynecol Oncol 2011 Jul;122(1):107-10.

5. Behbakht K, Randall TC, Benjamin I, Morgan MA, King S, Rubin SC. Clinical characteristics of clear cell carcinoma of the ovary. Gynecol Oncol 1998 Aug;70(2):255-8.

6. Sugiyama T, Kamura T, Kigawa J, Terakawa N, Kikuchi Y, Kita T, et al. Clinical characteristics of clear cell carcinoma of the ovary: a distinct histologic type with poor prognosis and resistance to platinum-based chemotherapy. Cancer 2000 Jun;88(11):2584-9.

7. Kobel M, Kalloger SE, Santos JL, Huntsman DG, Gilks CB, Swenerton KD. Tumor type and substage predict survival in stage I and II ovarian carcinoma: insights and implications. Gynecol Oncol 2010 Jan;116(1):50-6.

8. Anglesio MS, Carey MS, Kobel M, Mackay H, Huntsman DG. Clear cell carcinoma of the ovary: a report from the first Ovarian Clear Cell Symposium, June 24th, 2010. Gynecol Oncol 2011 May 1;121(2):407-15.

9. Skirnisdottir I, Seidal T, Karlsson MG, Sorbe B. Clinical and biological characteristics of clear cell carcinomas of the ovary in FIGO stages I-II. Int J Oncol 2005 Jan;26(1):177-83.

10. Campbell IG, Russell SE, Choong DY, Montgomery KG, Ciavarella ML, Hooi $\mathrm{CS}$, et al. Mutation of the PIK3CA gene in ovarian and breast cancer. Cancer Res 2004 Nov 1;64(21):7678-81.

11. Miyazawa M, Yasuda M, Fujita M, Kajiwara H, Hirabayashi K, Takekoshi S, et al. Therapeutic strategy targeting the mTOR-HIF-1alpha-VEGF pathway in ovarian clear cell adenocarcinoma. Pathol Int 2009 Jan;59(1):19-27.

12. Kobayashi $\mathrm{H}$, Kajiwara $\mathrm{H}$, Kanayama S, Yamada Y, Furukawa N, Noguchi $\mathrm{T}$, et al. Molecular pathogenesis of endometriosis-associated clear cell carcinoma of the ovary (review). Oncol Rep 2009 Aug;22(2):233-40.

13. Tan DS, Lambros MB, Rayter S, Natrajan R, Vatcheva R, Gao Q, et al. PPM1D is a potential therapeutic target in ovarian clear cell carcinomas. Clin Cancer Res 2009 Apr 1;15(7):2269-80.

14. Zorn KK, Bonome T, Gangi L, Chandramouli GV, Awtrey CS, Gardner GJ, et al. Gene expression profiles of serous, endometrioid, and clear cell subtypes of ovarian and endometrial cancer. Clin Cancer Res 2005 Sep 15;11(18):6422-30.

15. Mackay HJ, Brady MF, Oza AM, Reuss A, Pujade-Lauraine E, Swart AM, et al. Prognostic relevance of uncommon ovarian histology in women with stage III/IV epithelial ovarian cancer. Int J Gynecol Cancer 2010 Aug 1;20(6):945-52.

16. Mizuno M, Kikkawa F, Shibata K, Kajiyama H, Ino K, Kawai M, et al. Longterm follow-up and prognostic factor analysis in clear cell adenocarcinoma of the ovary. J Surg Oncol 2006 Aug 1;94(2):138-43.

17. Takano M, Sugiyama T, Yaegashi N, Sakuma M, Suzuki M, Saga Y, et al. Low response rate of second-line chemotherapy for recurrent or refractory clear cell carcinoma of the ovary: a retrospective Japan Clear Cell Carcinoma Study. Int J Gynecol Cancer 2008 Sep;18(5):937-42.

18. del Carmen MG, Birrer M, Schorge JO. Clear cell carcinoma of the ovary: a review of the literature. Gynecol Oncol 2012 Sep;126(3):481-90.

19. Rauh-Hain JA, Davis M, Clemmer J, Clark RM, Growdon WB, Goodman AK, et al. Prognostic determinants in patients with uterine and ovarian clear cell carcinoma: a SEER analysis. Gynecol Oncol 2013 Nov;131(2):404-9.

20. Crotzer DR, Sun CC, Coleman RL, Wolf JK, Levenback CF, Gershenson DM. Lack of effective systemic therapy for recurrent clear cell carcinoma of the ovary. Gynecol Oncol 2007 May;105(2):404-8.

21. Kajiyama H, Shibata K, Mizuno M, Yamamoto E, Fujiwara S, Umezu T, et al. Postrecurrent oncologic outcome of patients with ovarian clear cell carcinoma. Int J Gynecol Cancer 2012 Jun;22(5):801-6. 